\title{
The Dependence of the Efficiency of Electrohydrodynamic Heat Exchanger on the Electric Conductivity of Liquid
}

\author{
Vladimir Chirkov \\ Member, IEEE \\ St. Petersburg State University \\ 7/9 Universitetskaya nab. \\ St. Petersburg, 199034, Russia \\ v.chirkov@spbu.ru
}

\author{
Ekaterina Rodikova \\ St. Petersburg State University \\ 7/9 Universitetskaya nab. \\ St. Petersburg, 199034, Russia \\ e.rodikova@2012.spbu.ru
}

\author{
Yury Stishkov \\ St. Petersburg State University \\ 7/9 Universitetskaya nab. \\ St. Petersburg, 199034, Russia \\ y.stishkov@spbu.ru
}

\begin{abstract}
Electrohydrodynamic (EHD) flows are of great interest due to their capability of intensifying the heat exchange. However, the electric conductivity of liquid is typically disregarded, and the model of unipolar injection is used. The paper studies the effect of liquid conductivity on the efficiency of EHD heat exchanger with charge formed by injection as well as field-enhanced dissociation. The investigation involved computer simulation of the complete set of EHD equations supplemented with that for the heat transfer. The range of low-voltage conductivity of working liquid, when electroconvection can be used for heat transfer enhancement, was estimated. The EHD heat exchanger based on the field-enhanced dissociation is shown to be very promising for dielectric liquids with heightened conductivity.
\end{abstract}

Index Terms-Charge injection, computer simulation, dielectric liquids, electroconvection, field-enhanced dissociation, heat transfer enhancement, space charge.

\section{INTRODUCTION}

Electrohydrodynamic (EHD) flows are of great interest, since they can be used to intensify heat exchange [1-6] and have many advantages. Namely, an EHD heat exchanger (EHDHE) features very low power consumption, practically unlimited operation life, the ability to operate in microgravity, and high efficiency at meso- and micro-scale as opposed to other approaches [7]. The above makes for the continuous increase in the number of research works on the topic; however, at present, there are actually no investigations with concurrent computer simulation and experiment, because the corresponding physical processes are highly complicated. Nevertheless, the development of numerical models of isothermal electroconvection allowed bringing the simulation results nearer to the quantitative level, and a number of investigations devoted to the comparison of experimental and numerical data appeared [8-11]. In view of the above, the use of the up-to-date simulation models for EHDHE computeraided design is a topical issue and can result in quantitative evaluation of the device performance. To accomplish the latter, one has to allow for a number of factors, which were separately touched upon as a subject in several papers: the effect of temperature on liquid properties [12], the 07628. dependence of the injection function on the electric field strength $[11,13]$, and the effect of electrical conductivity of liquid on the injection EHDHE [14]. The latter issue is one of the most topical, and the paper is devoted to its investigation.

On the one hand, the effect of low-voltage conductivity $\left(\sigma_{0}\right)$ on the intensity of EHD flow was studied as early as the previous century [15], and the maximum velocity was shown to decrease at $\sigma_{0}>10^{-8} \mathrm{~S} / \mathrm{m}$. On the other hand, the role of dissociation-recombination processes in the bulk are ignored whereas actual liquids show finite (non-zero) conductivity level that has to affect the intensity and structure of the flow. Besides, one cannot help noting the mirror-like problem in the investigation of EHD conduction pumping when the emergence of the injection reverses the net flow direction. The issue is investigated by the group of Prof. Yagoobi [16].

Finally, there is a quite different aspect of the electric conductivity, namely, the field-enhanced dissociation or the so-called Wien effect [17]. Previously, the charge formation mechanism was ignored by most researchers into EHD flows and believed to be a purely theoretical phenomenon. Recently, however, the situation changed due to the immediate experimental evidence that intensive EHD flows emerge due to the Wien effect $[10,18]$. Moreover, the flow structure is rather similar to that of the injection electroconvection [19]. Therefore, the research into the effect of liquid conductivity on EHDHE performance has two sides: 1) the influence of dissociation-recombination processes on the injection electrohydrodynamic flow and 2) the possibility to design a new-type EHDHE basing on the Wien effect.

\section{MATHEMATICAL AND COMPUTER MODELS}

The investigation involved the computer simulation of the complete set of EHD equations [15, 20] supplemented with the heat transfer one:

$$
\begin{aligned}
& \operatorname{div}(\boldsymbol{E})=\rho / \varepsilon \varepsilon_{0} \\
& \boldsymbol{E}=-\nabla \varphi \\
& \partial n_{i} / \partial t+\operatorname{div}\left(\boldsymbol{j}_{i}\right)=W_{0} F(p)-\alpha_{r} n_{1} n_{2} \\
& \boldsymbol{j}_{i}=n_{i} b_{i} \boldsymbol{E}-D_{i} \nabla n_{i}+n_{i} \boldsymbol{u} \\
& W_{0}=\sigma_{0}^{2} /\left(e\left(\left|b_{1}\right|+\left|b_{2}\right|\right) \varepsilon \varepsilon_{0}\right)
\end{aligned}
$$




$$
\begin{aligned}
& \alpha_{\mathrm{r}}=e\left(\left|b_{1}\right|+\left|b_{2}\right|\right) /\left(\varepsilon \varepsilon_{0}\right) \\
& \rho=e\left(n_{1}-n_{2}\right) \\
& \gamma \partial \boldsymbol{u} / \partial t+\gamma(\boldsymbol{u}, \nabla) \boldsymbol{u}=-\nabla P+\eta \Delta \boldsymbol{u}+\rho \boldsymbol{E} \\
& \operatorname{div}(\boldsymbol{u})=0 \\
& \gamma C_{p} \partial T / \partial t+\operatorname{div}\left(-k \nabla T+\gamma C_{p} \boldsymbol{u} T\right)=0 \\
& F(p)=I_{1}(2 p) /(p) \\
& p=e^{2} /\left(k_{B} T\right) \sqrt{ }\left(E /\left(4 \pi \varepsilon \varepsilon_{0} e\right)\right),
\end{aligned}
$$

where $\boldsymbol{E}$ is the electric field strength, $\rho$ is the space charge density, $\varphi$ is the electric potential, $n$ is the ion concentration, $\boldsymbol{j}$ is the ion flux density, $\boldsymbol{u}$ is the fluid velocity, $P$ is the pressure, $T$ is the temperature, $\varepsilon$ is the relative electric permittivity, $\gamma$ is the mass density, $\eta$ is the dynamic viscosity, $b$ is the ion mobility, $D$ is the diffusion coefficient, $C_{p}$ is the specific heat at constant pressure, $k$ is the thermal conductivity coefficient; $W_{0}$ is the dissociation intensity, $\alpha_{r}$ is the recombination coefficient, $\varepsilon_{0}$ is the vacuum permittivity, $e$ is the elementary electric charge, $k_{B}$ is the Boltzmann constant, $t$ is the time; subscript $i$ indicates the ion species; $I_{1}$ is the modified Bessel function of the first kind; $F(p)$ is the Onsager function [17]. Ions are assumed to be monovalent. The set of equations is written for isothermal (and incompressible) liquid dielectric though the actual fluid properties are temperature dependent. However, the corresponding issue is beyond the scope of the present study, and the dependences are omitted to simplify the analysis. Some relevant information can be found in [12]. Besides, the model disregards the buoyancy force (since the EHDHE can operate even in the microgravity) and heat radiation (as it is a separate process).

Computations used software package COMSOL Multiphysics ${ }^{\circledR}$ based on the finite element method. A bladeplane electrode system was chosen for the simulation due to the following. Firstly, it features highly non-uniform electric field distribution, which in turn promotes the emergence of both the injection and field-enhanced dissociation mechanisms of charge formation. Secondly, the system (unlike that with a point electrode) can be used to cool an extended heat source. At last, it is a quite common design of EHDHE, which is used in a number of experimental and simulation works $[8,22-25]$. The geometry of computer model and the boundary conditions for the set of equations are given in Fig. 1. The lower plane doubles as the grounded electrode and heater simultaneously whereas the upper one is the cooler. It is worth noting that the blade is represented as a realistic surface rather than an injecting line/point at a plane (e.g., like it is in [24, 26]). This allows setting the injection charge formation at an area around the tip (e.g., like it is in $[13,25])$ and thus avoiding the problem with very steep change in space charge density near the injecting point. All computations were for a half (the right-hand one) of the cell due to the reflection symmetry, with the corresponding part being given at all two-dimensional plots below. The spatial

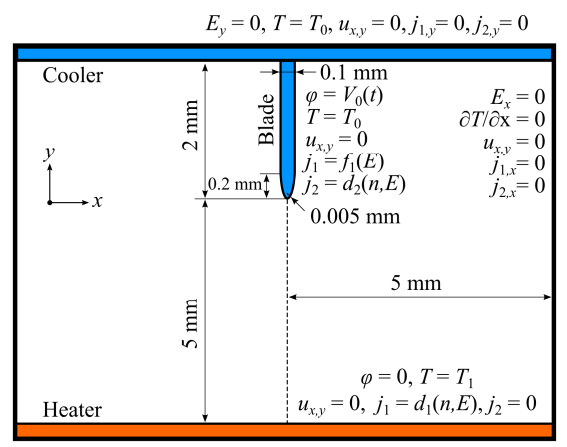

Fig. 1. Geometry of computer model and boundary conditions for the equation set.

size was chosen so as to be similar to that of a typical microprocessor, i.e., several millimeters. The natural convection is much less efficient comparing to EHD at such a spatial scale due to high friction losses. The temperature drop between the heater and the cooler, $\Delta T$, is $50^{\circ} \mathrm{C}$, a typical value of overheat for microprocessors. Besides, since liquid properties are independent of temperature, the results remain qualitatively the same, with the total heat flux being linearly proportional to the overheat value, when the latter varies. The voltage across the gap is reasonably set as great as possible but below the limit of cell breakdown strength, since the efficiency of heat removal increases with the voltage. Here, it is $25 \mathrm{kV}$.

The choice of the injection function is a separate issue that is very complicated and under active consideration at present $[11,13,21,23,27]$. Mainly, researchers who simulate EHD flows use autonomous injection (see, e.g., the review [21]). However, such an approach precludes taking into account the variations of injection current density with voltage and along the electrode surface as well as obtaining the current-voltage characteristic. A better way is using some kind of functional dependence of the injection current density on the electric field strength. Thus, the linear function is used here with coefficients being chosen to ensure the agreement on the order of magnitude between the total simulated current and experimental values for a cell with similar geometry $[11,23$, 28]:

$$
f_{1}(E)=A_{1}\left(E-E_{s t}\right) \cdot \vartheta\left(E-E_{s t}\right),
$$

where $A_{1}$ is the factor allowing for the intensity of the surface charge formation $\left(A_{1}=4 \times 10^{9} 1 /\left(\mathrm{m}^{2} \cdot \mathrm{s}\right)\right), E_{s t}$ is the suggested threshold value of the injection onset $\left(E_{s t}=5 \times 10^{6} \mathrm{~V} / \mathrm{m}\right), \vartheta(E)$ is the Heaviside step function. The charge loss is believed to be equal to the total current density for ions arriving to the boundary from the bulk:

$$
d_{i}(n, E)=n_{i} b_{i} E_{N}-D_{i} \nabla_{N} n_{i}
$$

where subscript $N$ denotes the normal to the surface of the electrode. In the first part of the next section, the injection charge formation at the blade surface and the charge loss at both electrodes are set whereas the Wien effect is disregarded. The latter is included into consideration instead of the injection in the second part of the results. 
The liquid properties correspond to those of transformer oil: $\varepsilon=2.2, \gamma=870 \mathrm{~kg} / \mathrm{m}^{3}, \eta=0.025 \mathrm{~Pa} \mathrm{~s},|b|=10^{-8} \mathrm{~m}^{2} /(\mathrm{V} \mathrm{s})$ (which is assumed to be the same for ions of both polarities), $D=1 \times 10^{-9} \mathrm{~m}^{2} / \mathrm{s}, k=0.18 \mathrm{~W} /(\mathrm{m} \mathrm{K}), C_{p}=2000 \mathrm{~J} /(\mathrm{kg} \mathrm{K})$. Low-voltage conductivity $\sigma_{0}$ is a parameter of study, and its value is varied (with other properties of the liquid left unchanged) in the range from $10^{-12}$ to $10^{-7} \mathrm{~S} / \mathrm{m}$.

The finite-element grid is constructed with allowance for features of unknown quantity distributions and has a mapped structure in the most important area (near both electrodes and within the central jet). The linear dimension of a finite element is about $1 \mu \mathrm{m}$ near the blade tip and $5 \mu \mathrm{m}$ near the heater surface. All computations continue until the steadystate regime is attained and then the equality of inward and outward thermal fluxes is verified.

\section{RESULTS AND DISCUSSION}

\section{A. Injection Electrohydrodynamic Heat Exchanger}

EHD flow structure in the blade-plane electrode system has been studied well enough in the case of the unipolar injection into initially non-conducting liquid (i.e., when $\sigma_{0}=$ $0 \mathrm{~S} / \mathrm{m}$ ). Ions emerge only at the blade tip and propagate to the counter electrode, this leads to the onset of an intensive electroconvection. The EHD jet strikes at the center of the plane electrode and spreads across its surface. If the latter is hotter than the liquid, the heat exchange takes place and the flow enhances the thermal flux to the cooler. Introducing a small conductivity into the model fails to change the described distributions when the concentration of injected ions is many times that of ions emerged due to the dissociation. The corresponding results (for $\sigma_{0}=10^{-11} \mathrm{~S} / \mathrm{m}$ ) are demonstrated in Fig. 2 where the distributions of space charge density, velocity with flow streamlines, and temperature with thermal flux lines are given. Fig. 2a shows the space charge to be propagating through the interelectrode gap without noticeable decrease in its magnitude; thus, the Coulomb force acts on the fluid within the whole gap providing high intensity of EHD flow (Fig. 2b).

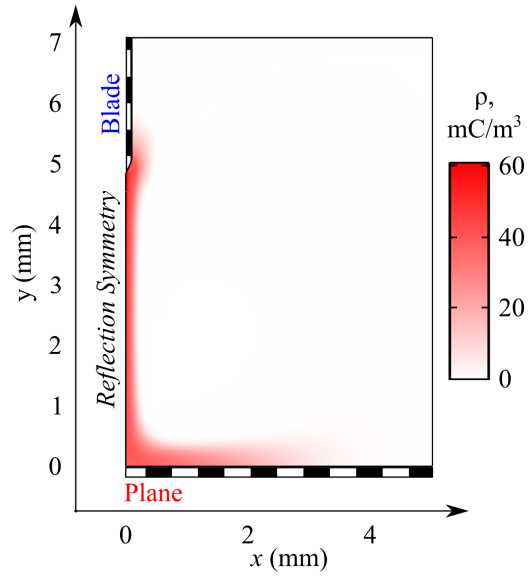

(a)

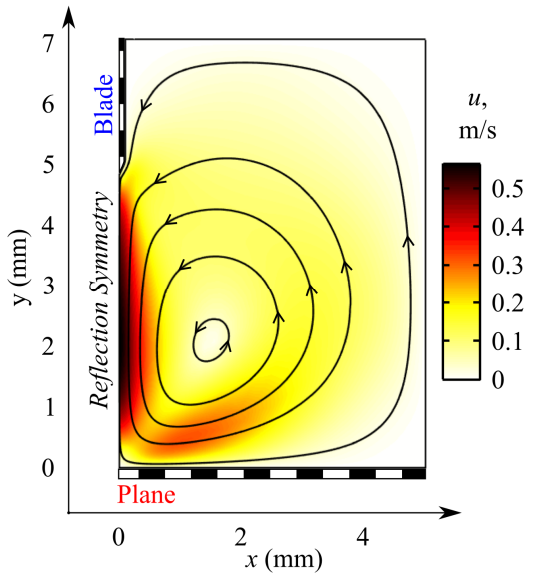

(b)
The downward flow of chilled liquid interacts with the heater, enhancing the heat flux from its surface and moving the warmed fluid to the cooler along the side face of the cell. As a result, the heat transfer within the bulk is provided mostly by electroconvection, with thermal flux lines (shown in Fig. 2c) nearly following fluid streamlines (Fig. 2b). The average heat flux density in the presented case is approximately 8 times the value that would be provided by the natural convection (at coefficient of thermal expansion $\left.\beta=6.5 \times 10^{-4} 1 / \mathrm{K}\right)$ in the same geometry $\left(3.3\right.$ vs. $\left.0.4 \mathrm{~W} / \mathrm{m}^{2}\right)$.

The increase in liquid conductivity leads to the enhancement of the injected-ion recombination during ion motion toward the counter electrode, which causes the Coulomb force to decrease as well as the area of its application to shorten. The main dimensionless parameter that evaluates the role of dissociation-recombination processes in the bulk (or the role of the conductivity) is the specific time scale ratio of two processes - ion motion $\left(\tau_{1}=L / u_{a}\right.$, where $u_{a}$ is the average fluid velocity) and charge relaxation $\left(\tau_{2}=\right.$ $\varepsilon \varepsilon_{0} / \sigma_{0}$ ). The mode change (i.e., $\tau_{1} / \tau_{2} \approx 1$ ) occurs during the increase in conductivity from $10^{-10}$ to $10^{-8} \mathrm{~S} / \mathrm{m}$ for typical electroconvection velocities. Besides, the conduction current density provided by negative ions starts to prevail over that of the injected positive ions near the blade surface in the conductivity range, which leads to the reversal of both the polarity of near-electrode layer and the EHD flow direction.

Fig. 3 gives the axial distributions of space charge density and $y$-component of fluid velocity (with the opposite sign) for three values of conductivity $\left(10^{-10}, 10^{-9}\right.$, and $\left.10^{-8} \mathrm{~S} / \mathrm{m}\right)$. Here, the positive value of fluid velocity corresponds to the downward direction of the central jet whereas the positive one- to the upward flow (along the axis). The electroconvection is directed toward the plane electrode in the first and second cases (curves 1 and 2 in Fig. 3b) when the injection current prevails over the conduction one, causing the homocharge to emerge near the blade electrode (curves 1 and 2 in Fig. 3a).

Nevertheless, the flow velocity diminishes along the axial path by an order of magnitude in the second case due to the

Fig. 2. Surface plots of space charge density (a), fluid velocity with flow streamlines (b), and temperature with thermal flux lines (c) in the injection EHDHE at $\sigma_{0}=10^{-11} \mathrm{~S} / \mathrm{m}$. 


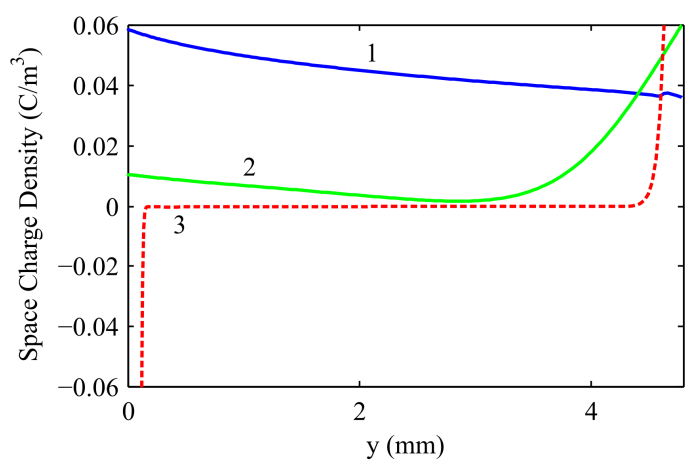

(a)

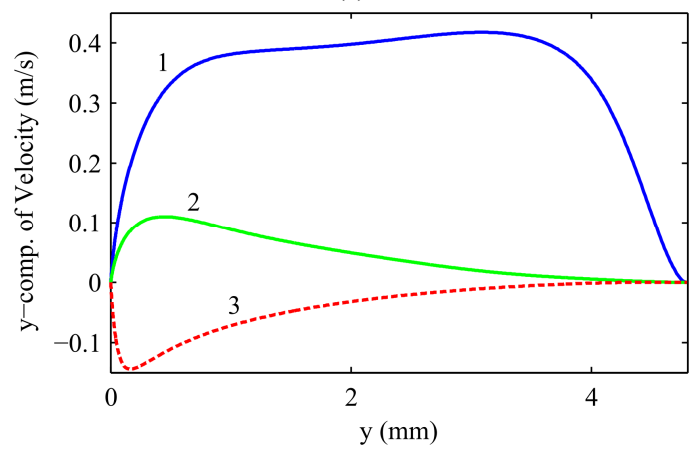

(b)

Fig. 3. Axial distributions of space charge density $(a)$ and y-component of fluid velocity $(b)$ in the injection EHDHE for three values of the conductivity $\left(1-10^{-10}, 2-10^{-9}\right.$, and $\left.3-10^{-8} \mathrm{~S} / \mathrm{m}\right)$.

space charge recombination. At last, the flow direction reverses when the injection current density fails to prevail over the conduction one, which causes heterocharge layers to emerge near both electrodes (curves 3 in Fig. 3a and b). All this leads to a considerable decrease in the average heat flux density from the hot plane as the conductivity of working liquid increases (curve 1 in Fig. 4); however, the heat removal reduces down to a non-zero value owing to the electroconvection-mode change from the ion-drag pumping to the conduction one. Some deviations of the approximation curve from the computed values are caused by the emergence of extra vortices in the bulk.

There is another important characteristic of EHDHE apart from the heat removal. It is the power consumption due to the Joule heating, which is of a negligible magnitude when liquid conductivity is low, but begins to play a significant role otherwise. Actually, a typical electric current value for the simulated EHD device (in the case of the injection into nonconducting liquid) lies below $0.1 \mu \mathrm{A}$ per 1 running $\mathrm{cm}$, with the power consumption being less than $1 \mathrm{~mW} / \mathrm{cm}$ (or $1 \mathrm{~mW} / \mathrm{cm}^{2}$ since the plane is $1 \mathrm{~cm}$ wide). However, the Joule heating becomes equal to the heat removal approximately at $\sigma_{0}=10^{-7} \mathrm{~S} / \mathrm{m}$ and begins to prevail over the latter at higher conductivity values (Fig. 4, curve 2). Thus, the natural limitation for the low-voltage conductivity level of the working liquid is $10^{-8}-10^{-7} \mathrm{~S} / \mathrm{m}$.

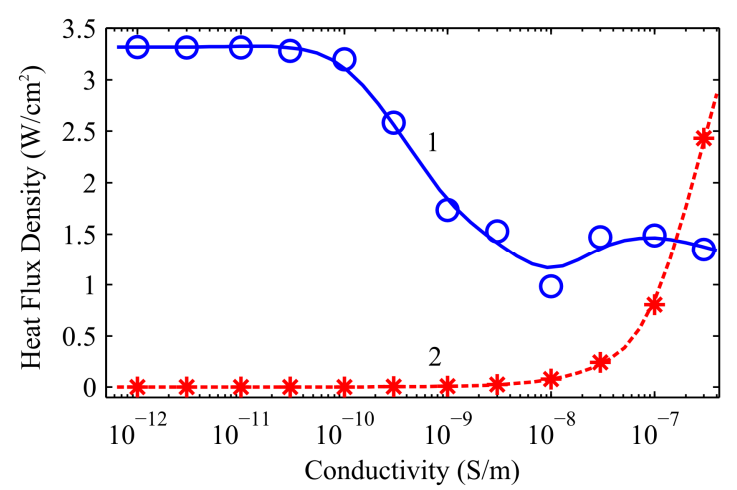

Fig. 4. The dependences of the average value of heat flux density (1) and power consumption (2) on the conductivity of liquid in the injection EHDHE; markers ("o" and "“*”) correspond to the computed values whereas curves are their approximation.

\section{B. Electrohydrodynamic Heat Exchanger Based on the Wien Effect}

At first sight, the above data seem to contradict the experimental papers (e.g., [8, 15, 29]), which show the existence of high speed EHD flows at comparably high conductivity values $\left(10^{-9}-10^{-8} \mathrm{~S} / \mathrm{m}\right)$. However, the flows are quite possible to be caused by the field-enhanced dissociation rather than the injection charge formation. Thus, references $[10,30]$ provide experimental evidence that the Wien effect leads to the emergence of intensive EHD flows; therefore, the charge-formation mechanism can underlie the EHDHE.

The space charge density and fluid velocity distributions together with electroconvection streamlines in the EHDHE based on the Wien effect are exemplified in Fig. 5. The structure is seen to be very similar to that observed in the injection heat exchanger (Fig. 2). Thus, the homocharge emerges in the interelectrode gap and, therefore, the fluid moves from the sharp electrode to the blunted one (unlike in standard conduction pumping). Moreover, the flow intensity is very high and the velocity (in the presented case) even exceeds $1 \mathrm{~m} / \mathrm{s}$.

Of course, the results strongly depend on the voltage and conductivity values due to the features of the Wien effect. The dependence of the maximum flow velocity on the conductivity is shown in Fig. 6. Despite a relative increase in the dissociation rate under the effect of the strong electric field being independent of the low-voltage conductivity, the space charge emerging in the bulk appears to be proportional to the value of the latter. Thus, the intensity of EHD flows in EHDHE based on the field-enhanced dissociation rises with the conductivity, and a steep increase in the maximum value of fluid velocity takes place in the conductivity range from $10^{-10}$ to $10^{-8} \mathrm{~S} / \mathrm{m}$ (unlike the case of the injection EHDHE). The corresponding fluid velocity reaches $1.5 \mathrm{~m} / \mathrm{s}$ and then falls at higher conductivity values due to the enhancement of space charge recombination. 


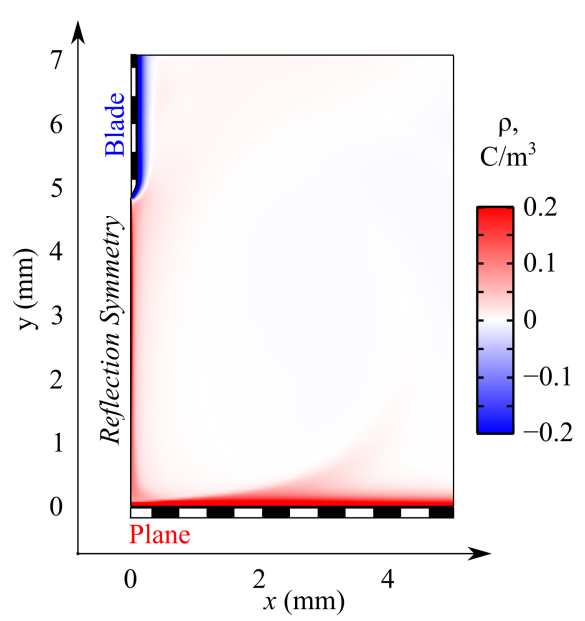

(a)

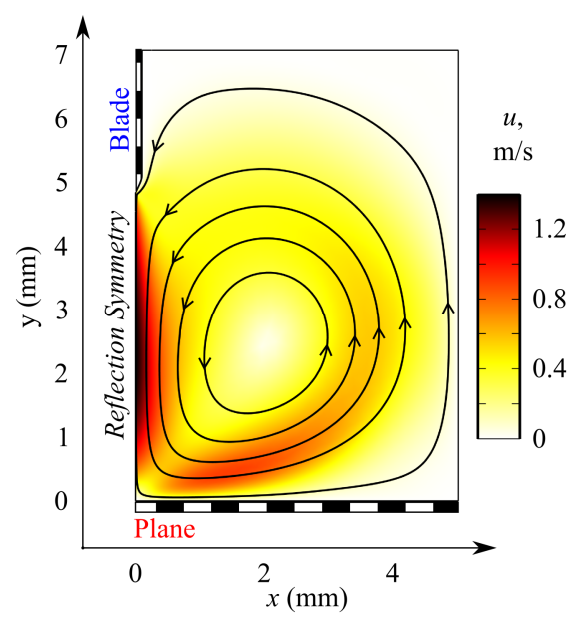

(b)

Fig. 5. Contour plot of space charge density (a) and fluid velocity with flow streamlines (b) at $\sigma_{0}=3 \cdot 10^{-9} \mathrm{~S} / \mathrm{m}$ in the EHDHE based on the Wien effect.

All this causes the increase in the efficiency within the studied conductivity range (curve 1 in Fig. 7), with the maximum value of heat removal being even higher than that obtained for the injection EHDHE. The power consumption due to the Joule heating is actually negligible as compared to the heat removal when the conductivity value is below $10^{-8} \mathrm{~S} / \mathrm{m}$ and becomes significant otherwise. Thus, the electroconvection emerging owing to the Wien effect is believed to be a very promising phenomenon to be utilized in

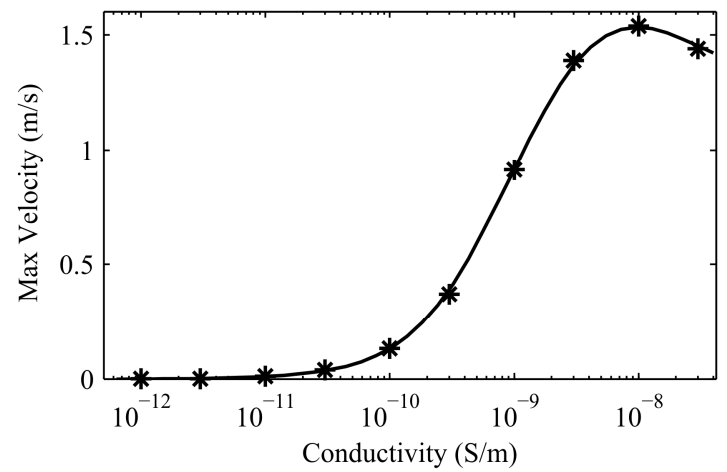

Fig. 6. The dependence of the maximum flow velocity on the conductivity of liquid in the EHDHE based on the Wien effect.

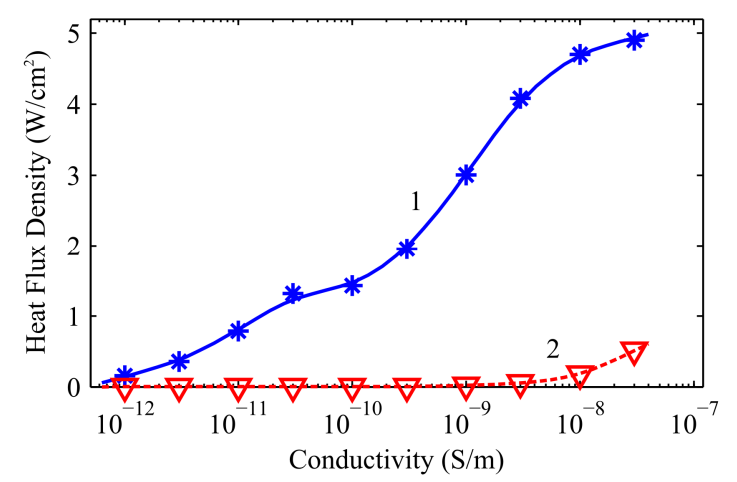

Fig. 7. The dependence of the average value of heat flux density (1) and power consumption (2) on the conductivity of liquid in the EHDHE based on the Wien effect; markers ("*” and " $\nabla$ ") correspond to the computed values whereas curves are their approximation. heat exchangers. Besides, a very important feature of the field-enhanced dissociation is worth noting. Namely, almost all information needed for the computer simulation of the EHDHE basing on the Wien effect is known, unlike the case of the injection charge formation where the injection law (and its temperature dependence) represents a very complicated issue.

\section{CONCLUSION}

The electric conductivity of the working liquid strongly affects the performance of EHD heat exchanger. Its increase leads to a steep decrease in the efficiency of heat transfer due to the lessening of the flow intensity when the injection charge formation underlies the emergence of electroconvection. However, the heat transfer enhancement can be ensured by virtue of the electroconvection caused by the Wien effect in the case of heightened liquid conductivity, with the efficiency of the corresponding EHD heat exchanger being comparable or even better than that of the injection one of the same geometry. Thus, the electroconvection can enhance heat transfer in a wide range of liquid conductivity; however, the prevailing charge formation mechanism has to be thoroughly analyzed when designing EHD heat exchanger.

\section{ACKNOWLEDGMENT}

Research was carried out using resources provided by the Computer Center of SPbU and Center for Diagnostics of Functional Materials for Medicine, Pharmacology and Nanoelectronics of Research park of St. Petersburg State University.

\section{REFERENCES}

[1] B. R. Lazarenko, F. P. Grosu, and M. K. Bologa, "Convective heattransfer enhancement by electric fields," Int. J. Heat Mass Transf., vol. 18, no. 12, pp. 1433-1441, 1975.

[2] M. K. Bologa, F. P. Grosu, and I. A. Kozhukhar', Electroconvection and Heat Transfer, Chisinau: Shtiintsa, 1977 (in Russian). 
[3] P. Atten, F. M. J. McCluskey, and A. T. Perez, "Electroconvection and its Effect on Heat Transfer," IEEE Trans. Electr. Insul., vol. 23, no. 4, pp. 659-667, 1988.

[4] F. M. J. McCluskey, P. Atten, and A. T. Perez, "Heat transfer enhancement by electroconvection resulting from an injected space charge between parallel plates," Int. J. Heat Mass Transf., vol. 34, no. 9, pp. 2237-2250, 1991.

[5] V. K. Patel, F. Robinson, J. Seyed-Yagoobi, and J. Didion, "Terrestrial and microgravity experimental study of microscale heat-transport device driven by electrohydrodynamic conduction pumping," IEEE Trans. Ind. Appl., vol. 49, no. 6, pp. 2397-2401, 2013.

[6] P. Traoré, A. Perez, D. Koulova, and H. Romat, "Numerical modelling of finite-amplitude electro-thermo-convection in a dielectric liquid layer subjected to both unipolar injection and temperature gradient," $J$. Fluid Mech., vol. 658, pp. 279-293, 2010.

[7] M. R. Pearson and J. Seyed-Yagoobi, "Experimental study of EHD conduction pumping at the meso- and micro-scale," J. Electrostat., vol. 69, no. 6, pp. 479-485, 2011.

[8] P. Traoré, M. Daaboul, and C. Louste, "Numerical simulation and PIV experimental analysis of electrohydrodynamic plumes induced by a blade electrode," J. Phys. D. Appl. Phys., vol. 43, pp. 1-8, 2010.

[9] L. Yang, K. S. Minchev, M. Talmor, C. Jiang, B. C. Shaw, and J. Seyed-Yagoobi, "Flow distribution control in meso scale via electrohydrodynamic conduction pumping," in Proc. 2015 IEEE Industry Applications Society Annual Meeting, pp. 1-8.

[10] V. A. Chirkov, D. K. Komarov, Y. K. Stishkov, and S. A. Vasilkov, "Comparative analysis of numerical simulation and PIV experimental results for a flow caused by field-enhanced dissociation," J. Phys. Conf. Ser., vol. 646, p. 012033, 2015.

[11] A. Gazaryan, A. Sitnikov, V. Chirkov, Yu. Stishkov, "A Method for Estimation of Functional Dependence of Injection Charge Formation on Electric Field Strength," in Proc. 2016 Electrostatics Joint Conf., pp. 1-8.

[12] J. Wu and P. Traoré, "A Finite-Volume Method for ElectroThermoconvective Phenomena in a Plane Layer of Dielectric Liquid," Numer. Heat Transf. Part A Appl., vol. 68, no. 5, pp. 471-500, 2015.

[13] P. Traore, J. Wu, C. Louste, Q. Pelletier, and L. Dascalescu, "Electrohydrodynamic Plumes due to Autonomous and Nonautonomous Charge Injection by a Sharp Blade Electrode in a Dielectric Liquid," IEEE Trans. Ind. Appl., vol. 51, no. 3, pp. 25042512, May 2015.

[14] V. Chirkov, E. Rodikova, Yu. Stishkov, "The Dependence of the Efficiency of Electrohydrodynamic Heat Exchanger on the Electric Conductivity of Liquid," in Proc. 2016 Electrostatics Joint Conf., pp. 1-8.

[15] Yu. K. Stishkov and A. A. Ostapenko, Electrohydrodynamical Flows in Liquid Dielectrics, Publishing House of Leningrad State Univ., Leningrad, 1989 (in Russian).
[16] M. Yazdani and J. S. Yagoobi, "The effect of uni/bipolar charge injection on EHD conduction pumping," J. Electrostat., vol. 75, pp. 43-48, Jun. 2015.

[17] L. Onsager, "Deviations from Ohm's law in weak electrolytes," J. Chem. Phys., vol. 2, pp. 599-615, 1934.

[18] J. C. Ryu, H. J. Park, J. K. Park, and K. H. Kang, "New electrohydrodynamic flow caused by the Onsager effect," Phys. Rev. Lett., vol. 104, no. 10, pp. 1-4, 2010.

[19] V. A. Chirkov, Y. K. Stishkov, and A. A. Sitnikov, "Integral electric current characteristics of unsteady-state processes of current passage through liquid dielectrics," IEEE Trans. Dielectr. Electr. Insul., vol. 22, no. 5, pp. 2763-2769, 2015.

[20] A. Castellanos, Electrohydrodynamics, Wien: Springer, 1998.

[21] Y. K. Suh, "Modeling and simulation of ion transport in dielectric liquids - Fundamentals and review," IEEE Trans. Dielectr. Electr. Insul., vol. 19, no. 3, pp. 831-848, 2012.

[22] A. I. Zhakin and A. E. Kuz'ko, "Electroconvective Heat Transfer across Boundary Layers," High Temp., vol. 39, no. 5, pp. 783-785, 2001.

[23] A. I. Zhakin and A. E. Kuzko, "Electrohydrodynamic flows and heat transfer in the blade-plane electrode system," Fluid Dyn., vol. 48, no. 3, pp. 310-320, May 2013.

[24] A. T. Pérez, P. Traoré, D. Koulova-Nenova, and H. Romat, "Numerical study of an electrohydrodynamic plume between a blade injector and a flat plate," IEEE Trans. Dielectr. Electr. Insul., vol. 16, no. 2, pp. 448455, Apr. 2009.

[25] J. Wu, P. Traore, C. Louste, D. Koulova, and H. Romat, "Direct numerical simulation of electrohydrodynamic plumes generated by a hyperbolic blade electrode," J. Electrostat., vol. 71, no. 3, pp. 326331, Jun. 2013.

[26] J. Wu, P. Traore, C. Louste, L. Dascalescu, F. Tian, and A. T. Perez, "Numerical Investigation of Electrohydrodynamic Plumes for Locally Enhanced Cooling in Dielectric Liquids," IEEE Trans. Ind. Appl., vol. 51, no. 1, pp. 669-678, Jan. 2015.

[27] A. I. Zhakin, "Near-electrode and transient processes in liquid dielectrics," Physics-Uspekhi, vol. 49, no. 3, pp. 275-295, 2006.

[28] Y. K. Stishkov, A. A. Sitnikov, and V. A. Chirkov, "The investigation of EHD flow structure in blade-plane electrode system using PIVtechnique," Surf. Eng. Appl. Electrochem., vol. 52, to be published.

[29] M. Daaboul, C. Louste, and H. Romat, "PIV measurements on charged plumes-influence of $\mathrm{SiO} 2$ seeding particles on the electrical behavior," IEEE Trans. Dielectr. Electr. Insul., vol. 16, no. 2, pp. 335-342, 2009.

[30] V. A. Chirkov, Y. K. Stishkov, S. A. Vasilkov, "PIV investigation of EHD flow caused by field-enhanced dissociation," in Proc. of 10th Conf. of the French Society of Electrostatics, 2016, pp. 1-4. 